\title{
NÍVEIS DE ADUBAÇÃO N E K EM CAFEEIROS FERTIRRIGADOS E SUA FUNÇÃO DE RESPOSTA
}

\author{
Gleice Aparecida Assis ${ }^{*}$, Mario Roberto Prata Melo ${ }^{2}$, Mara Alves Soares ${ }^{1}$, Franscinely Aparecida de \\ Assis $^{3}$, William Eduardo dos Reis Martins ${ }^{4}$, Lucas Rafael Silva ${ }^{4}$ \\ ${ }^{1}$ Docente da Universidade Federal de Uberlândia - Campus Monte Carmelo, Monte Carmelo - MG \\ ${ }^{2}$ Mestrando em Ciência e Tecnologia Ambiental na Universidade Federal do Triângulo Mineiro, Uberaba - MG \\ ${ }^{3}$ Docente efetiva da UniCerrado - Centro Universitário de Goiatuba, Goiatuba - GO \\ ${ }^{4}$ Discente do Curso de Agronomia da Universidade Federal de Uberlândia - Campus Monte Carmelo, Monte Carmelo - MG \\ *Autora para correspondência: Gleice Aparecida de Assis, gleice@ufu.br
}

\begin{abstract}
RESUMO: O cafeeiro irrigado exige um manejo diferenciado de fertilizantes em relação à lavoura de sequeiro, devido a disponibilidade de água constante durante 0 ano. Nesse sentido, há a necessidade do desenvolvimento de pesquisas para que se possa promover a otimização dos fertilizantes, fato que faz das funções de produção importante instrumento nos estudos econômicos das produções agrícolas. Com isso, objetivou-se neste trabalho estimar a função de produção, visando determinar o nível de adubação nitrogenada e potássica economicamente viáveis para a produção de cafeeiro fertirrigado na região do Cerrado Mineiro. $O$ experimento foi conduzido na Fazenda Juliana, Monte Carmelo, em Minas Gerais. O plantio foi realizado em novembro de 2011 utilizando-se mudas da cultivar Topázio MG 1190. Foram testados cinco níveis de $\mathrm{N}$ (Nitrogênio) e $\mathrm{K}_{2} \mathrm{O}$ (Potássio) (30, 80, 130, 180 e 230\%) do recomendado para lavouras não irrigadas. Verificou-se que a produtividade física máxima de 43,6 sacas ha ${ }^{-1}$ foi atingida com nível de adubação de $178,14 \%$ da recomendação para sequeiro. Entretanto, o nível de adubação ótimo, com que se obteve maior retorno econômico foi de 141,35\%, resultando em uma produtividade de 41,88 sacas ha-1, o que demonstra uma diferença de $36,79 \%$ entre as eficiências técnica e econômica. Considerando-se a eficiência econômica, houve uma redução de gastos de $\mathrm{R} \$ 377,34$ ha-1 e R $\$$ 784,00 ha $^{-1}$, respectivamente, com o uso de adubo nitrogenado e potássico.
\end{abstract}

PALAVRAS-CHAVE: Coffea arabica L., viabilidade econômica, demanda nutricional

\section{LEVELS OF NITROGEN AND POTASSIUM FERTILIZATION IN COFFEE FERTIGATED AND RESPONSE FUNCTION}

\begin{abstract}
Irrigated coffee trees require differentiated management of fertilizers in relation to dry farming, due to the constant availability of water during the year. There is a need for the development of research to promote the optimization of fertilizers, a fact that makes production functions an important instrument in the economic studies of agricultural production. Thus, this study aimed to estimate the production function to determine the level of nitrogen and potassium fertilization economically feasible to produce fertirrigated coffee trees in Minas Gerais's Cerrado region. The experiment was conducted at Juliana Farm, in the municipality of Monte Carmelo, Minas Gerais. Seedlings of the "Topázio MG 1190" cultivar were planted in November 2011. Five levels of nitrogen and potassium 30,80,130, 180 and 230\%, the recommendation for non-irrigated crops were tested. It was verified that the maximum physical productivity of 43.6 bags ha- $^{-1}$ was reached with a fertilization level of $178.14 \%$, the recommendation for dry farming. However, the optimum level of fertilizer with the highest economic return was $141.35 \%$, resulting in a yield of 41.88 bags ha $^{-1}$, which shows a $36.79 \%$ difference between the technical and economic efficiency. Regarding the economic efficiency, there were reduced expenses of BRL 377.34 ha $^{-1}$ and BRL 784.00 ha $^{-1}$ with the use of nitrogen and potassium fertilizers, respectively.
\end{abstract}

KEYWORDS: Coffea arabica L., economic feasibility, nutritional demand 


\section{INTRODUÇÃO}

O cafeeiro (Coffea spp.) é uma cultura de grande importância econômica e social para 0 Brasil. 0 país se destaca por ser 0 maior produtor e exportador dessa commodity, estimando-se para a safra de 2018 uma produção de 58 milhões de sacas de 60 quilos de café beneficiado (CONAB, 2018).

Minas Gerais é responsável por $63,2 \%$ da produção nacional, destacando-se neste estado o cultivo da espécie Coffea arabica L. (CONAB, 2018). A região do Cerrado Mineiro, englobando o Triângulo Mineiro, Alto Paranaíba e Noroeste, se destaca por ser a primeira do país a receber a Denominação de Origem, cujo objetivo é agregar qualidade e valor ao produto, tornando-o bastante competitivo no agronegócio e valorizado pelo mercado externo.

Apesar do sucesso da implantação da cafeicultura no Cerrado Mineiro, apresenta limitação em relação a disponibilidade hídrica, sendo a irrigação fundamental para o êxito da produtividade. Até mesmo em regiões aptas à cultura, como o sul de Minas, a irrigação é válida, pois oferece benefícios, tanto na fase de formação (Sobreira et al., 2011) quanto de produção (Scalco et al., 2011). A cafeicultura irrigada ocupa $10 \%$ da área total de café cultivado no Brasil, aproximadamente 240 mil hectares, sendo responsável por $25 \%$ da produção de café no país, permitindo classificar o cafeeiro entre as principais culturas irrigadas do cenário agrícola brasileiro (Fernandes et al., 2012).

A aplicação de nutrientes via água de irrigação, técnica denominada de fertirrigação, vem sendo amplamente utilizada nas lavouras cafeeiras (Villela et al., 2015). Atualmente essa técnica é utilizada sem um padrão especifico de recomendação para lavouras irrigadas, utilizando a recomendação para lavouras de sequeiro, podendo tal fato comprometer 0 desenvolvimento das plantas devido a falta ou excesso de nutrientes, sendo que o cafeeiro irrigado apresenta padrões de crescimento e produtividade diferentes do não irrigado (Carvalho et al., 2006; Rezende et al., 2010; Silva et al., 2008). Baixas produtividades em cafeeiros irrigados têm sido estreitamente relacionadas à desordens nutricionais devido à falta de um programa de manejo de adubação (Assis et al., 2015).

Outro fato que deve ser considerado no manejo da adubação exclusivamente via água de irrigação é que o custo dos fertilizantes específicos para essa técnica leva a uma relação benefício-custo menor que a adubação convencional, o que justifica o desenvolvimento de pesquisas que possam auxiliar na redução da quantidade aplicada de fertilizantes, gerando economia para os cafeicultores.

Neste contexto, surgem as funções de produção, que têm sido importante instrumento nos estudos econômicos das produções agrícolas. Conhecidos os preços dos insumos e do produto, pode-se determinar a quantidade ótima de cada insumo a ser empregado para que a lucratividade do produtor rural seja maximizada (Pereira, 2005).

De acordo com Frizzone et al. (1995) a função de resposta ou de produção das culturas é uma relação física entre as quantidades de certo conjunto de insumos e as quantidades físicas máximas que podem ser obtidas do produto, para dada tecnologia. Assim, mediante a necessidade da redução de custos para o manejo da fertirrigação cafeeira, objetivou-se neste trabalho determinar a função de produção, visando avaliar o nível de adubação nitrogenada e potássica economicamente viável para a produção de cafeeiro fertirrigado na região do Cerrado Mineiro.

\section{MATERIAL E MÉTODOS}

O experimento foi conduzido na Fazenda Juliana, no município de Monte Carmelo, em Minas Gerais, com as seguintes coordenadas: latitude $18^{\circ} 43^{\prime} 29^{\prime \prime} \mathrm{S}$, longitude $47^{\circ} 29^{\prime} 55^{\prime \prime}$ W e altitude de $870 \mathrm{~m}$

O plantio da lavoura foi realizado em novembro de 2011 utilizando-se mudas de Coffea arabica L., cultivar Topázio MG 1190. Foi adotado espaçamento de 3,8 m entre linhas e 0,6 m entre plantas, totalizando 4.386 plantas ha $^{-1}$. Na tabela 1 é apresentada a caracterização química do solo nos anos de 2012, 2013 e 2014 
Tabela 1. Caracterização química do solo (camada 0 - 20cm) em 2012, 2013, 2014 e 2015.

\begin{tabular}{|c|c|c|c|c|}
\hline Característica & 2012 & 2013 & 2014 & 2015 \\
\hline $\mathrm{pH}\left(\mathrm{H}_{2} \mathrm{O}\right)$ & 6,1 & 6,3 & 5,5 & 6,8 \\
\hline Fósforo $(P)-\mathrm{mg} \mathrm{dm}^{-3}$ & 10,5 & 62,4 & 4,4 & 7,4 \\
\hline Potássio $(\mathrm{K})-\mathrm{mg} \mathrm{dm}^{-3}$ & 69,0 & 222,0 & 74,0 & 110 \\
\hline Cálcio $\left(\mathrm{Ca}^{2+}\right)-\mathrm{cmolc} \mathrm{dm}^{-3}$ & 2,6 & 6,0 & 1,4 & 3,3 \\
\hline Magnésio $\left(\mathrm{Mg}^{2+}\right)-\mathrm{cmolc} \mathrm{dm}^{-3}$ & 1,8 & 1,1 & 0,5 & 1,6 \\
\hline Alumínio $\left(\mathrm{Al}^{3+}\right)-\mathrm{cmolc} \mathrm{dm}^{-3}$ & 0,55 & 0,0 & 0,0 & 0,0 \\
\hline $\mathrm{H}+\mathrm{Al}\left(\right.$ Extrator SMP) - cmolc $\mathrm{dm}^{-3}$ & 2,5 & 1,8 & 3,3 & 1,9 \\
\hline $\mathrm{SB}-\mathrm{cmolc} \mathrm{dm}^{-3}$ & 4,5 & 7,6 & 2,0 & 5,21 \\
\hline CTC (t) - cmolc dm ${ }^{-3}$ & 5,1 & 7,6 & 2,1 & 5,21 \\
\hline CTC a pH 7,0(T) - cmolc dm ${ }^{-3}$ & 7,0 & 9,4 & 5,3 & 7,11 \\
\hline $\mathrm{V}-\%$ & 65,0 & 81,0 & 39,0 & 73 \\
\hline$m-\%$ & 11,0 & 0,0 & 3,0 & 0 \\
\hline Matéria orgânica $(M O)$ - dag kg-1 & 2,3 & 3,4 & 1,8 & 2,4 \\
\hline Zinco $(\mathrm{Zn})-\mathrm{mg} \mathrm{dm}^{-3}$ & 1,9 & 3,8 & 1,0 & 1,6 \\
\hline Ferro $(\mathrm{Fe})-\mathrm{mg} \mathrm{dm}^{-3}$ & 41,0 & 31,0 & 49,0 & 48 \\
\hline Manganês (Mn) - $\mathrm{mg} \mathrm{dm}^{-3}$ & 5,4 & 8,9 & 3,2 & 6,0 \\
\hline Cobre $(\mathrm{Cu})-\mathrm{mg} \mathrm{dm}^{-3}$ & 0,2 & 3,8 & 0,3 & 0,3 \\
\hline Boro (B) - mg dm-3 & 0,14 & 0,5 & 0,3 & 0,29 \\
\hline Enxofre $(\mathrm{S})-\mathrm{mg} \mathrm{dm}^{-3}$ & 23,0 & 216,0 & 5,0 & 2,0 \\
\hline
\end{tabular}

Fonte: Laboratório Brasileiro de Análises Agrícolas (Labras).

Foram estudados cinco níveis de adubação e 3 são apresentadas, respectivamente, a quantidade nitrogenada e potássica (30,80,130, 180 e $230 \%$ de de adubo aplicado (ureia pecuária e nitrato de potássio) $\mathrm{N} \mathrm{e} \mathrm{K}_{2} \mathrm{O}$ ) do recomendado para lavouras não irrigadas, e a quantidade de nutriente ( $\left.\mathrm{N} \mathrm{e} \mathrm{K}_{2} \mathrm{O}\right)$ em 2014 e 2015, segundo recomendação de Guimarães et al. (1999), com base na análise de solo, e ainda na Tabela 4 a aplicados neste estudo via fertirrigação. Nas tabelas 2 produtividade nestes dois anos.

Tabela 2. Quantidade de adubo (Kg ha-1 ano-$\left.^{-1}\right)$ aplicada em cada tratamento.

\begin{tabular}{ccccc}
\hline & \multicolumn{3}{c}{2014} & \multicolumn{2}{c}{2015} \\
\cline { 2 - 5 } Níveis de adubação(a) & Ureia pecuária & Nitrato de potássio & Ureia pecuária & Nitrato de potássio \\
\hline $30 \%$ & 152,28 & 153,42 & 202,8 & 204,5 \\
$80 \%$ & 406,08 & 409,12 & 540,7 & 545,2 \\
$130 \%$ & 659,88 & 664,82 & 878,7 & 886,3 \\
$180 \%$ & 913,68 & 920,52 & 1216,7 & 1227,2 \\
$230 \%$ & 1167,48 & 1176,22 & 1554,7 & 1568,1 \\
\hline
\end{tabular}

(a) Níveis de adubação baseados na recomendação de Guimarães et al. (1999).

Tabela 3. Quantidade de $\mathrm{Ne} \mathrm{K}_{2} \mathrm{O}\left(\mathrm{Kg} \mathrm{ha}^{-1} \mathrm{ano}^{-1}\right)$ aplicada em cada tratamento.

\begin{tabular}{ccccccc}
\hline \multirow{2}{*}{ Níveis de adubação(a) } & \multicolumn{2}{c}{2014} & \multicolumn{2}{c}{2015} & \multicolumn{3}{c}{ Médias } \\
\cline { 2 - 7 } & $\mathrm{N}$ & $\mathrm{K}_{2} \mathrm{O}$ & $\mathrm{N}$ & $\mathrm{K}_{2} \mathrm{O}$ & $\mathrm{N}$ & $\mathrm{K}_{2} \mathrm{O}$ \\
\hline $30 \%$ & 88,5 & 67,5 & 117,8 & 90,0 & 103,1 & 78,7 \\
$80 \%$ & 235,9 & 180,0 & 314,2 & 239,9 & 275,0 & 209,9 \\
$130 \%$ & 383,4 & 292,5 & 510,6 & 390,0 & 447,0 & 341,2 \\
$180 \%$ & 530,8 & 405,0 & 707,0 & 540,0 & 618,9 & 472,5 \\
$230 \%$ & 678,3 & 517,5 & 903,5 & 690,0 & 790,9 & 603,7 \\
\hline Adubação padrão de sequeiro & 295,0 & 225,0 & 393,0 & 300,0 & 344,0 & 262,0 \\
\hline
\end{tabular}

(a) Níveis de adubação baseados na recomendação de Guimarães et al. (1999). 
Tabela 4. Produtividade média do cafeeiro (sacas ha-1) em 2014 e 2015 em função dos níveis de adubação utilizados no experimento.

\begin{tabular}{rc}
\hline Níveis de adubação & Médias \\
\hline $30 \%$ & 13,97 \\
$80 \%$ & 37,70 \\
$130 \%$ & 35,75 \\
$180 \%$ & 43,97 \\
$230 \%$ & 40,80 \\
\hline
\end{tabular}

Fonte: Dados da pesquisa

Para adubação foram utilizadas fontes solúveis de nutrientes indicados para a fertirrigação: ureia pecuária ( $45 \%$ de $\mathrm{N})$ e nitrato de potássio $(44 \%$ de $\mathrm{K}_{2} \mathrm{O}$ e $13 \%$ de N). O parcelamento da adubação foi realizado da seguinte forma: nos meses de novembro a abril, as plantas receberam $70 \%$ do total aplicado no ano; e de maio a outubro $30 \%$ da adubação total. Em novembro de 2014 foi realizada aplicação de $1,17 \mathrm{t} \mathrm{ha}^{-1}$ de calcário dolomítico com poder relativo de neutralização de 85\%. Em 2015 não foi realizada aplicação de corretivos, pois o solo se encontrava com saturação por bases de 73\% (Tabela 1). Em função do baixo teor de fósforo no solo em 2014 (4,4 mg dm-3) foi realizada aplicação de $55 \mathrm{~kg}$ de $\mathrm{P}_{2} \mathrm{O}_{5} \mathrm{ha}^{-1}$, utilizando-se como fonte o fosfato monoamônico (44\% de $\mathrm{P}_{2} \mathrm{O}_{5}$ e $9 \%$ de $\mathrm{N}$ ).

O manejo fitossanitário foi realizado mediante avaliações periódicas na lavoura para determinação da necessidade de manejo de pragas, doenças e plantas daninhas. Para o controle de plantas daninhas em préemergência aplicou-se herbicida a base de oxifluorfen na dose de $2 \mathrm{~L}$ ha-1 (p.c.), além de capinas manuais periódicas na projeção da copa do cafeeiro. Para o controle de cercosporiose (Cercospora coffeicola Berk. \& Cooke) e Mancha de Phoma (Phoma costarricensis Echandi) aplicou-se, com auxílio de bomba costal, fungicidas à base de tiofanato metílico, na dose de $1,0 \mathrm{~kg} \mathrm{ha}^{-1}$ (p.c.) e vazão de $400 \mathrm{~L} \mathrm{ha-1}$. Em relação à ferrugem do cafeeiro (Hemileia vastatrix Berk \& Br.) utilizou-se fungicidas dos grupos químicos dos triazois e estrobirulinas, tais como o ciproconazol e azoxistrobina, na dose de $500 \mathrm{~mL} \mathrm{ha}^{-1}$ (p.c.). 0 controle de bicho-mineiro-do-cafeeiro (Leucoptera coffeella Guérin-Mèneville) foi realizado com clorpirifós, na dose de $1,5 \mathrm{~L} \mathrm{ha}^{-1}$ (p.c.).

0 delineamento experimental utilizado foi o de blocos casualizados, com quatro repetições e cinco tratamentos, totalizando 20 parcelas experimentais.
Cada unidade experimental foi constituída por 10 plantas, sendo oito úteis. Entre cada linha de tratamento de plantas irrigadas existiam duas linhas de plantas de bordadura, de forma a evitar uma possível interferência de um tratamento de adubação sobre o outro.

Foi realizada a colheita no mês de julho nos anos de 2014 e 2015, medindo-se a produção de café das oito plantas centrais de cada parcela experimental. A colheita foi realizada quando restaram, no máximo, $20 \%$ de frutos verdes, por meio de derriça manual no pano. Após a determinação do volume, foi retirada uma amostra de 10 litros que foi colocada para secar em terreiro suspenso. Após atingir a umidade de $11 \%$ foram determinados a massa e o volume do café em coco.

Posteriormente, as amostras foram beneficiadas e novamente foram determinados a massa, o volume e a umidade. A relação do volume inicial colhido no pano, o volume da amostra de 10 litros do café colhido no pano e a massa da amostra já beneficiada forneceu a produtividade em quilo de cada parcela, que foi extrapolada para sacas ha-1, em função do espaçamento adotado na lavoura. A produtividade total de cada tratamento (sacas ha-1 ${ }^{-1}$ foi resultante da média da produtividade do café colhido na planta nos anos de 2014 e 2015.

Dentre os modelos polinomiais existentes, a função quadrática é uma das mais usadas para o ajuste de uma função de produção por apresentar retornos crescentes, ponto de máximo ou mínimo e retornos decrescentes, o que é esperado para o uso de diferentes doses de fertilizantes (Carvalho, 1992).

Para obtenção da função de produção, foi utilizada a análise de regressão entre a variável dependente (produtividade) e a variável independente (níveis de adubação). 0 modelo empregado foi o polinomial do segundo grau, conforme expressão (1). 
$y=a+b x+c x$

Onde:

$y=$ produtividade de café beneficiado (sacas ha-1 ${ }^{-1}$; $x=$ nível de adubação nitrogenada e potássica (\%);

$a, b, c=$ parâmetros da equação.

O nível de adubação nitrogenada e potássica de maior retorno econômico a ser aplicado deve corresponder a uma produtividade que otimize a lucratividade do experimento. 0 produto físico marginal (PFMa) foi obtido derivando-se a função polinomial de segundo grau da produtividade do café beneficiado, e, em seguida, este indicador técnico foi igualado à relação econômica de preços entre o fator nível de adubação nitrogenada e potássica e o produto café beneficiado (em sacas), para obtenção do nível de adubação ótimo, que reflete a máxima eficiência econômica, como descrito na expressão (2):

$\frac{\partial_{y}}{\partial_{x}}=b-2 c x=\frac{P_{x}}{P_{y}}$

Onde:

$\frac{\partial_{y}}{\partial_{x}}=\begin{aligned} & \text { derivada da função polinomial de segundo grau } \\ & \text { da produtividade. }\end{aligned}$

$\frac{P_{x}}{P_{y}}=\begin{aligned} & \text { relação de preços entre o nível de adubação e } 0 \\ & \text { produto café beneficiado. }\end{aligned}$

$b, c, x=$ coeficientes da função.

O PFMa do fator variável é o aumento no produto físico total decorrente do emprego de uma unidade adicional do fator variável. Graficamente, o PFMa representa a inclinação do produto total ou da função de produção em um determinado nível do fator variável, ou seja, representado por uma linha tangente à curva de resposta, e é um dos indicadores das eficiências técnica e econômica do experimento.

Da expressão (2), obtém-se o indicador de eficiência econômica conforme a expressão (3).

$\frac{\partial_{y}}{\partial_{x}}=\frac{P_{x}}{P_{y}}$

A obtenção do nível de adubação nitrogenada e potássica que proporciona máximo retorno econômico foi feito por meio da derivada parcial, igualando ao preço do fator variável. Com os valores conhecidos de preço de venda do produto (Py - em saca de café beneficiado) e do preço da adubação nitrogenada e potássica referente à $1 \%$ da recomendação de adubação proposta por Guimarães et al. (1999) foi calculado o nível de adubação que proporcionou maior rendimento econômico.

Os preços da saca de café beneficiado no período de 2013 e 2014, assim como o valor dos adubos utilizados nos anos de 2013 e 2014 são apresentados na Tabela 5. Os preços são cotados em datas diferentes do ano de produção pelo fato de que a produção de uma safra é garantida pela adubação do ano anterior à ela. Por ser o café um produto voltado para o mercado externo, procurou-se trabalhar com a relação dos preços em dólar. Na Tabela 6 é apresentado o preço médio mensal do dólar durante o período de produção e comercialização do café.

Tabela 5. Preço mensal da saca de $60 \mathrm{Kg}$ de café beneficiado tipo 6 e preço médio do $\mathrm{Kg}$ de $\mathrm{N}$ e K2O no período de 2013 e 2014 (em dólar).

\begin{tabular}{ccccccccc}
\hline \multirow{2}{*}{ Mês } & Ano & \multicolumn{9}{c}{2013} & \multicolumn{4}{c}{2014} \\
\cline { 2 - 9 } & Saca & $\mathrm{N}$ & $\mathrm{K}_{2} \mathrm{O}$ & Total $\left(\mathrm{N}+\mathrm{K}_{2} \mathrm{O}\right)$ & Saca & $\mathrm{N}$ & $\mathrm{K}_{2} \mathrm{O}$ & Total $\left(\mathrm{N}+\mathrm{K}_{2} \mathrm{O}\right)$ \\
\hline Jan. & 163,49 & 1,49 & 4,03 & 5,52 & 115,33 & 1,34 & 3,14 & 4,48 \\
Fev. & 156,48 & 1,53 & 4,62 & 6,15 & 142,68 & 1,11 & 4,29 & 5,4 \\
Mar. & 151,97 & 1,45 & 2,43 & 3,88 & 185,20 & 1,28 & 3,89 & 5,17 \\
Abr. & 148,82 & 1,44 & 2,60 & 4,04 & 193,21 & 1,35 & 4,08 & 5,43 \\
Mai & 150,82 & 1,43 & 2,59 & 4,02 & 181,83 & 1,35 & 4,20 & 5,55 \\
Jun. & 130,72 & 1,32 & 4,08 & 5,40 & 172,37 & 1,34 & 4,18 & 5,52 \\
Jul. & 132,13 & 1,30 & 3,94 & 5,24 & 169,27 & 1,35 & 4,18 & 5,53 \\
Ago. & 124,29 & 1,23 & 3,78 & 5,01 & 175,89 & 1,32 & 3,74 & 5,06 \\
Set. & 121,96 & 1,23 & 3,58 & 4,81 & 170,38 & 1,29 & 3,66 & 4,95 \\
Out. & 113,40 & 1,29 & 3,73 & 5,02 & 187,05 & 1,23 & 3,26 & 4,49 \\
Nov. & 102,47 & 1,25 & 3,28 & 4,53 & 182,75 & 1,18 & 3,12 & 4,3 \\
Dez. & 107,92 & 1,22 & 3,20 & 4,42 & 177,33 & 1,14 & 3,02 & 4,16 \\
\hline Média & 133,70 & 1,35 & 3,49 & 4,84 & 171,10 & 1,27 & 3,73 & 5,00 \\
\hline
\end{tabular}

Fonte: Cooperativa Regional de Cafeicultores em Guaxupé (Cooxupé). 
Tabela 6. Valor em reais do dólar mensal médio.

\begin{tabular}{ccc}
\hline Mês & 2013 & 2014 \\
\hline Jan. & 2,03 & 2,39 \\
Fev. & 1,97 & 2,39 \\
Mar. & 1,98 & 2,34 \\
Abr. & 2,01 & 2,23 \\
Mai. & 2,02 & 2,22 \\
Jun. & 2,18 & 2,23 \\
Jul. & 2,25 & 2,23 \\
Ago. & 2,34 & 2,26 \\
Set. & 2,28 & 2,32 \\
Out. & 2,19 & 2,44 \\
Nov. & 2,29 & 2,55 \\
Dez. & 2,34 & 2,63 \\
Média & 2,16 & 2,35 \\
\hline
\end{tabular}

Fonte: Cooxupé.

Os dados foram submetidos à análise de variância pelo software estatístico Sisvar (Ferreira, 2011), sendo posteriormente realizada análise de regressão para o fator quantitativo (níveis de adubação).

\section{RESULTADOS E DISCUSSÃO}

Houve efeito significativo $(p<0,01)$ dos níveis de adubação para a média de produtividade de café beneficiado em 2014 e 2015.

Por se tratar de um fator quantitativo, foi aplicada análise de regressão para o fator níveis de adubação. A escolha do modelo polinomial baseou-se no nível de significância $(p<0,01)$ e no maior coeficiente de determinação $\left(R^{2}\right)$.

Observou-se que a função quadrática (Figura 1) apresentou ajuste satisfatório aos dados $\left(R^{2}=88 \%\right)$. A partir desse modelo fica evidente que a produtividade atinge o máximo de 43,6 sacas ha-1 utilizando-se um nível de adubação de 178,14\%. Após atingir esse máximo, a produtividade tende a diminuir em relação ao aumento dos níveis mais elevados de adubação, indicando um possível desequilíbrio nutricional (Malavolta, 1993; Quintela et al., 2011).

Figura 1. Produtividade de café beneficiado (sacas de $60 \mathrm{~kg} \mathrm{ha}^{-1}$ ) sob níveis de adubação nitrogenada e potássica.

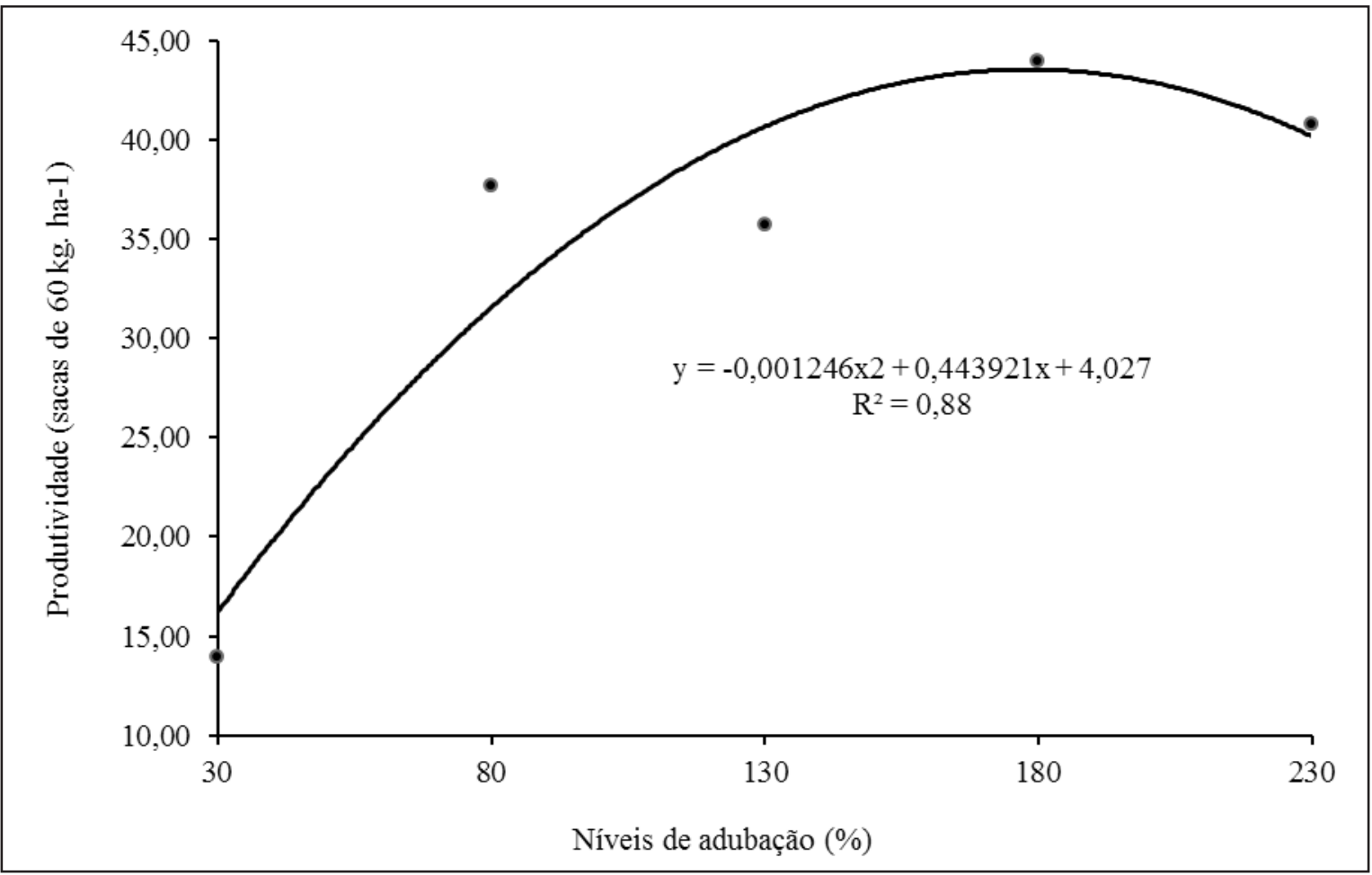


Resultados semelhantes foram observados por Assis et al. (2015), a qual verificou que a utilização de $171,11 \%$ da dose recomendada para lavouras de sequeiro proporcionou a máxima produtividade física de 84,82 sacas ha ${ }^{-1}$. Foi verificado também que a partir da dose de $130 \%$ da recomendada para cafeeiro não irrigado houve uma tendência de estabilização da produtividade com 0 aumento da adubação.

Entretanto, Sobreira et al. (2011) ao estudarem o parcelamento e a dose de nitrogênio e potássio mais adequado para o primeiro ano de formação da lavoura adensada de cafeeiro fertirrigado, observaram que a adubação via irrigação poderia ser reduzida em $30 \%$ da recomendação proposta por Guimarães et al. (1999). Os fatores atribuídos a suficiência da dose de 70\% foram o alto nível de fertilidade inicial do solo levando à falta de resposta, principalmente da adubação potássica; a lavoura ter sido conduzida de forma adensada e as plantas estarem na fase de formação, não apresentando um dreno forte de nutrientes como ocorre em cafeeiro na fase produtiva.

Derivando-se a função da produtividade do café beneficiado, foi obtido o produto físico marginal (PFMa) e, em seguida, igualando-se este indicador técnico à relação econômica de preços entre fator adubação e produto café beneficiado, foi obtido o nível de adubação que proporcionou o máximo retorno econômico, como descrito na expressão (2).

Observa-se, na Tabela 7, que o PFMa é inicialmente positivo e decresce à medida que se aumenta o nível de adubação nitrogenada e potássica aplicada ao solo, mantendo-se negativo a partir do ponto máximo da curva de resposta.

Tabela 7. Produto físico marginal para os níveis de adubação nitrogenada e potássica.

\begin{tabular}{cc}
\hline Níveis de adubação (\%) & Produto físico marginal \\
\hline 30 & 0,369161 \\
80 & 0,244561 \\
130 & 0,119961 \\
180 & $-0,004639$ \\
230 & $-0,129239$ \\
\hline
\end{tabular}

De acordo com Lima Júnior et al. (2011), quando o valor do PFMa atinge o valor zero, significa que 0 nível de adubação nitrogenada e potássica aplicado proporcionou a produtividade máxima do experimento, ou seja, a máxima eficiência técnica dos fatores mantidos constantes no experimento, sendo obtida igualando-se a primeira derivada a zero (PFMa $=0$ ), representando 43,6 sacas ha-1, aplicando um nível de adubação de $178,14 \%$ do recomendado por Guimarães et al. (1999).

A partir do ponto em que o PFMa apresentou valor nulo, a aplicação de um maior nível de adubação conduziu ao valor de produto físico marginal negativo, indicando ser antieconômico o uso dessa quantidade de adubo para as condições tecnológicas utilizadas neste experimento, o que indica uma superutilização dos fatores mantidos fixos na área experimental.

O nível ótimo de adubação foi calculado igualando-se a expressão do PFMa à relação de preços do fator variável $(\mathrm{Px})$ no nível de adubação de $1 \%$ do recomendado para lavouras cafeeiras e do produto (Py), expresso em sacas de café beneficiado, como expresso na equação (4), obtendo-se, dessa forma, a máxima eficiência econômica. Assim, o nível de adubação com que se obteve maior retorno econômico foi de $141,35 \%$, resultando em uma produtividade de 41,88 sacas ha-1. Esse nível de adubação corresponde à aplicação de $486,24 \mathrm{~kg}$ de $\mathrm{N} \mathrm{ha}^{-1}$ e $370,34 \mathrm{~kg}$ de $\mathrm{K}_{2} \mathrm{O}$ ha $^{-1}$. Fazendo-se uma análise comparativa com a recomendação de adubação proposta por Guimarães et al. (1999) para lavouras cafeeiras não irrigadas, verifica-se que para uma produtividade esperada de 40 a 50 sacas ha-1 e considerando um baixo teor de nitrogênio na folha $\left(<2,5 \mathrm{dag} \mathrm{kg}^{-1}\right)$ e baixo teor de potássio no solo $\left(<60 \mathrm{mg} \mathrm{dm}^{-3}\right)$, a recomendação é de $350 \mathrm{~kg}$ de $\mathrm{N} \mathrm{e} \mathrm{K}_{2} \mathrm{O}$ ha-1. Por se tratar de uma lavoura fertirrigada, com potencial de produção superior à uma lavoura de sequeiro, é justificável esse aumento na demanda de nutrientes.

$$
\frac{\partial_{y}}{\partial_{x}}=-0,00249 x+0,443921=\frac{P_{x}}{P_{y}}
$$

Ao comparar o nível de adubação ótimo e o que proporcionou a máxima produtividade física, 
verifica-se valores bem contrastantes, expressando uma diferença de $36,79 \%$ entre a eficiência econômica e a eficiência técnica. Assim, pode-se afirmar que seria possível uma economia de $126,5 \mathrm{~kg}$ de $\mathrm{N}^{-1} \mathrm{r}^{-1}$ e 96,4 $\mathrm{kg}$ de $\mathrm{K}_{2} \mathrm{O} \mathrm{ha}^{-1}$ ao adubar o cafeeiro com o nível de adubação de 141,35\%, quando comparado à 178,14\% (correspondente a uma adubação de $612,8 \mathrm{~kg}$ de $\mathrm{N} \mathrm{ha}^{-1}$ e 466,73 ${\mathrm{kg} \text { de } \mathrm{K}_{2} \mathrm{O} \mathrm{ha}}^{-1}$ ).

Dessa forma, o produtor estaria economizando US\$ 165,71 (R\$ 377,34) no adubo nitrogenado por hectare (considerando o preço do quilo de nitrogênio a US\$1,31) e US\$348 (R\$784,00) no adubo potássico por hectare (considerando o preço do quilo de potássio a US\$ 3,61).

Como já visto, apesar de uma determinada dose de fertilizante ser responsável por proporcionar a máxima produtividade de uma cultura, pode ser que esta não seja aquela que garanta ao produtor o máximo de retorno econômico. Carvalho (1992), buscando determinar o nível de adubação que seria responsável pela maior lucratividade em uma lavoura de café situada no sul de Minas Gerais, encontrou que as doses mais econômicas de nitrogênio e potássio a serem aplicados no cafeeiro foram de 189,38 e 203,99 Kg ha 1, respectivamente, menores que a recomendação do IBC (Instituto Brasileiro de Café) para sequeiro, onde as doses mais viáveis foram de $300 \mathrm{Kg} \mathrm{ha}^{-1}$ de $\mathrm{N}$ e 450 $\mathrm{Kg} \mathrm{ha}^{-1}$ de $\mathrm{K}_{2} \mathrm{O}$.

Diante do exposto conclui-se que o nível de adubação ótimo, com que se obteve maior retorno econômico foi de $141,35 \%$ da adubação recomendada de nitrogênio e potássio, resultando em uma produtividade de 41,88 sacas ha-1 ${ }^{-1}$ o que demonstra uma diferença de $36,79 \%$ entre as eficiências técnica e econômica. Considerando-se a eficiência econômica, houve uma redução de gastos de $R \$ 377,34$ ha- $^{-1}$ e $R \$ 784,00$ ha $^{-1}$, respectivamente, com 0 uso de adubo nitrogenado e potássico no cafeeiro fertirrigado.

\section{REFERÊNCIAS BIBLIOGRÁFICAS}

Assis, G.A.; Guimarães, R. J.; Colombo, A.; Scalco, M.S.; Dominghetti, A.W. Critical ranges for leaf nitrogen and potassium levels in coffee fertigated at the production phase. Revista Ciência Agronômica, 2015, v. 46, n. 1, p. 126-134.
Carvalho, C.H.M. de.; Colombo, A.; Scalco, M.S.; Morais, A.R. Evolução do crescimento do cafeeiro (Coffea arabica L.) irrigado e não irrigado em duas densidades de plantio. Ciência e Agrotecnologia, 2006, v. 30 , n. 2 , p. $243-250$.

Carvalho, F.M. de. A estratégia dos produtores de café com relação ao uso econômico de nitrogênio e potássio, município de Lavras - MG. Lavras: UFLA, (Dissertação - Mestrado em Administração Rural). 1992. 67 p.

Companhia Nacional de Abastecimento - CONAB. Acompanhamento da safra brasileira de café, 2018, v. 5 - Safra 2018, n. 2 - Segundo levantamento, Brasília, p. 1-66. Disponível em: <https://www.conab.gov.br/index. php/info-agro/safras> Acesso em 21 de Maio de 2018.

Cooperativa Regional de Cafeicultores em Guaxupé. Preço Histórico do Café. 2015. Disponível em: $\quad<h t t p: / / p o r t a l w e b . c o o x u p e . c o m . b r: 8080 /$ portal/ precohistoricocafe.jsp>. Acesso em: 20 nov. 2015.

Fernandes, A.L.T.; Partelli, F.L.; Bonomo, R.; Golynski, A. A moderna cafeicultura dos cerrados brasileiros. Pesquisa Agropecuária Tropical, 2012, v. 42, n. 2, p. 231-240.

Ferreira, D.F. Sisvar: um sistema computacional de análise estatística. Ciência e Agrotecnologia, 2011, v.35, n.6, p.1039-1042.

Frizzone, J.A.; Teodoro, R.E.F.; Pereira, A.S.; Botrel, T.A. Lâminas de água e doses de nitrogênio na produção de aveia (Avena sativa L.) para forragem. Scientia Agrícola, 1995, v. 52, n. 3, p. 578-586.

Guimarães, P.T.G.; Garcia, A.W.R.; Alvarez, V.H.; Prezotti, L.C.; Viana, A.S.; Miguel, A.E.; Malavolta, E.; Corrêa, J.B.; Lopes, A.S.; Nogueira, F.D.; Monteiro, A.V.C. In: COMISSÃO DE FERTILIDADE DO SOLO DO ESTADO DE MINAS GERAIS - CFSEMG. Recomendações para o uso de corretivos e fertilizantes em Minas Gerais, $5^{\circ}$ Aproximação. Editores, RIBEIRO et al., Viçosa, MG, 1999, 359 p. 289-302.

Lima Junior, J.A.de.; Pereira, G.M.; Geisenhoff, L.O.; Costa, G.G.; Reis, R.P.; Oliveira, L.F.C. Avaliação econômica da produção de alface americana em função de lâminas de irrigação. Ciência e Agrotecnologia, 2011, v. 35 , n. 2, p.392-398. 
Malavolta, E. Nutrição mineral e adubação do cafeeiro. São Paulo: Agronômica Ceres. 1993. p. 64-126.

Pereira, J.R.D. Viabilidade técnica e econômica das aplicações de água e nitrogênio no cultivo de gladíolo (Gladiolus $\mathrm{x}$ grandiflorus L.). Tese (Doutorado em Engenharia Agrícola) - Universidade Federal de Lavras, Lavras - MG. 2005. 80 p.

Quintela, M.P.; Silva, T.J.A.; Bonfim-Silva, E.M.; Silva, E.F.F.; Bebé, F.V. Parâmetros produtivos e nutricionais do cafeeiro submetido adubação nitrogenada na região de Garanhuns. Revista Caatinga, 2011, v. 24, n. 4, p. 74-79.

Rezende, F.C.; Helbel Júnior, C.; Souza, R.S. de; Antunes, F.M.; Frizzone, J.A. Crescimento inicial de duas cultivares de cafeeiro em diferentes regimes hídricos e dosagens de fertirrigação. Engenharia Agrícola, 2010, v. 30, n. 3, p. 447-458.
Scalco, M.S.; Alvarenga, L.A.; Guimarães, R.J.; Colombo, A.; Assis, G.A. Cultivo irrigado e não irrigado do cafeeiro (Coffea arabica L.) em plantio superadensado. Coffee Science, 2011, v. 6, n. 3, p. 193-202.

Silva, C.A. da,; Teodoro, R.E.F.; Melo, B.de. Produtividade e rendimento do cafeeiro submetido a lâmina de irrigação. Pesquisa Agropecuária Brasileira, 2008 , v. 43 , n. 3, p. 387-394.

Sobreira, F.M.; Guimarães, R.J.; Colombo, A.; Scalco, M.S.; Carvalho, J.G. Adubação nitrogenada e potássica de cafeeiro fertirrigado na fase de formação, em plantio adensado. Pesquisa Agropecuária Brasileira, 2011, v. 46, n. 1, p.9-16.

Villela, G.M.; Guimarães, R.J.; Pinto, C.G.; Scalco, M.S.; Sales Junior, J.C.; Camilo, W.R.; Alves, G. Faixas críticas de teores foliares de macronutrientes para cafeeiros fertirrigados em formação. Coffee Science, 2015 , v. 10 , n. 3 , p. $271-279$. 